\title{
Valve sparing-aortic root replacement with the reimplantation technique in acute type $A$ aortic dissection
}

\author{
Stefano Mastrobuoni, Laurent De Kerchove, Emiliano Navarra, Parla Astarci, Philippe Noirhomme, \\ Gebrine El Khoury \\ Department of Cardiovascular and Thoracic Surgery, St. Luc'S Hospital, Catholic University of Louvain, Brussels, Belgium \\ Correspondence to: Stefano Mastrobuoni. Cardiovascular And Thoracic Surgery, St. Luc'S Hospital, Avenue Hippocrate 10, 1200 Brussels, \\ Belgium. Email: stefano.mastrobuoni@uclouvain.be.
}

Submitted Jan 06, 2016. Accepted for publication Apr 12, 2016.

doi: $10.21037 /$ acs.2016.04.04

View this article at: http://dx.doi.org/10.21037/acs.2016.04.04

\section{Clinical vignette}

A 56-year-old man presented to the emergency department of our institution complaining of sudden onset chest pain, shortness of breath and palpitations. His past medical history was significant for hypertension and paroxysmal atrial fibrillation. Physical examination revealed a blood pressure of $150 / 80 \mathrm{mmHg}$, heart rate of $120 \mathrm{bpm}$, normal heart sounds and lungs auscultation and normal distal arterial pulses. An urgent computed tomography (CT) scan of the chest and abdomen revealed an acute type A aortic dissection extending from the ascending aorta till the origin of the superior mesenteric artery with a maximum diameter of $51 \mathrm{~mm}$ at the level of the ascending aorta. The patient was therefore referred for emergency surgery.

\section{Surgical techniques}

\section{Preparation}

Standard approach with patient under general anesthesia in the supine position, mechanical ventilation, internal jugular central venous line, bilateral radial artery cannulation, transesophageal echo, axillary temperature and Foley catheter.

\section{Exposition}

Our standard approach is to expose the right axillary artery before sternotomy and to cannulate this vessel for the cardio-pulmonary bypass using a $10 \mathrm{~mm}$ Dacron graft if the patient is stable. Otherwise we pursue a femoral vessels cannulation. The sternum is then opened in standard fashion, the right atrium is cannulated and cardiopulmonary bypass (CPB) is started cooling the body temperature to $25^{\circ} \mathrm{C}$.

\section{Operation}

During cooling the brachiocephalic trunk and the left common carotid artery are dissected and prepared for clamping. A left ventricular vent is introduced trough the right superior pulmonary vein. At $25^{\circ} \mathrm{C}$, the $\mathrm{CPB}$ is stopped, the brachiocephalic trunk is clamped and selective antegrade cerebral perfusion is started via the axillary cannula and a cannula inserted directly into the left common carotid artery. Cold crystalloid cardioplegia is also given through the coronary ostia for myocardial protection. The aortic root and valve are then assessed to confirm the indication for valve sparing aortic root replacement (VSRR). At this stage, the sizing of the Valsalva graft used for the reimplantation technique can be carried out (see below). The distal portion of the same graft can be eventually used for aortic arch replacement.

Reconstruction of the distal aorta is performed first with total or partial aortic arch replacement using a straight Dacron graft. The dissected aortic layers are bond together using some BioGlue ${ }^{\circledR}$ (Cryolife Inc, Kennesaw, GA, USA) and reinforced with Teflon felt between the layers and/ or externally. Once the distal anastomosis is completed, the cerebral perfusion is stopped and the aorta is de-aired. The Dacron graft is then clamped and systemic perfusion is restarted as well as rewarming.

The surgical steps towards the reimplantation technique 
are described below and presented in the video (Video 1).

\section{Root exposure and preparation}

The aortic root is prepared for VSRR procedure. First, three commissural traction stitches are placed at the tip of each commissure. If dissected, the aortic root layers are glued to facilitate the next steps including external root preparation, resection of the sinuses of Valsalva and creation of coronary buttons.

\section{Root dissection}

From this stage, the reimplantation technique is very similar to the technique described in cases of aortic root aneurism. The root is separated from the surrounding structure down to the level of ventriculo-aortic junction. In case of acute dissection, the adventitia can be difficult to recognize as this tissue is often thickened by a diffuse hematoma. However, care must be taken to leave this adventitial layer on the aortic side especially behind the commissures because this tissue is necessary to reinforce the media which alone may not be strong enough to resist the wall stress. Also, it is important to keep the adventitial tissues around the coronary buttons to reinforce them and provide better hemostasis.

Once the root is free, the sinuses of Valsalva are resected leaving approximately 5 to $8 \mathrm{~mm}$ of aortic rim. The right and left coronary buttons are created leaving a generous patch of aortic wall. During the course of the reimplantation technique, warm blood cardioplegia is given intermittently through the coronary Ostia. The cannulas are fixed to the coronary buttons in order to give the cardioplegia while the surgeon can continue the procedure.

\section{Proximal suture line}

Nine to twelve 2-0 Tycron stitches with pledget are generally used for the proximal suture line. They are distributed along the circumference of the ventriculo-aortic junction. Sutures are passed from inside the aorta to outside with the pledgets on the inside, starting from the noncoronary/left coronary (NC/LC) commissure and moving clockwise. Along the fibrous portion of the aortic annulus, these sutures are inserted along the horizontal plane formed by the base of the inter-leaflet triangles. Notably however, along the non-fibrous portions of the annulus where the external dissection of the aortic root is limited by muscle these sutures are inserted along the lowest portion of the freely dissected aortic root making the proximal suture line slightly higher at the RC/NC and RC/LC commissures compared to the $\mathrm{LC} / \mathrm{NC}$ commissure.

\section{Graft preparation and fixation}

A Dacron prosthesis with built-in neo-aortic sinuses (GelweaveValsalva ${ }^{\mathrm{TM}}$ graft, Vascutek Ltd, a Terumo company, Renfrewshire, Scotland) is generally used. The graft sizing is based on the height of the commissure between the nonand the left coronary sinuses. The height is measured with a ruler from the base of interleaflet triangle to the top of the commissure. This measurement corresponds to the size of the graft that will be used. When this measurement does not correspond to a labelled graft size, the next larger size graft is chosen. The most common graft sizes range between 28 and $32 \mathrm{~mm}$.

Due to the anatomy of the base of the aortic root, the proximal sutures are not in a linear plane and the proximal end of the graft need to be trimmed accordingly. The pledgeted sutures are then passed through the base of the prosthesis, respecting the spacing and importantly the curvilinear contour of the proximal suture line. Finally the Dacron graft is pulled down and the stitches are tied to ensure appropriate seating of the graft around the aortic annulus.

\section{Valve reimplantation}

The commissures are reimplanted first using 4-0 polypropylene sutures. To prevent valve distortion, the three commissures must be reattached at the same level of the neo-sinotubular junction of the Valsalva graft. Radial traction is then applied on two adjacent commissural sutures to help delineate the 'line of implantation'. A running suture starting from the commissure and aimed to fix the rest of the valve to the graft is then performed in small, regular steps passing the suture from outside the prosthesis to inside and through the aortic wall, staying close to the annulus, and then back out of the prosthesis.

\section{Valve testing and leaflet management}

After valve reimplantation, it is critical to re-examine the leaflets for any residual prolapse, symmetry, and the height and depth of coaptation. After placing the leaflets into their closure position, a sealing test is carried out using a syringe of saline solution flushed with pressure in the neo-aortic root. The valve is then re-observed after suction of the same solution.

\section{Coronary reimplantation}

The left coronary button is re-implanted first. A hole 
of approximately 6 to $8 \mathrm{~mm}$ of diameter is made in the graft in face of the coronary button. Reimplantation is performed with a running suture of 5-0 polypropylene. A strip of autologous pericardium can be used to reinforce the anastomosis and improve the hemostasis. A similar technique is used for reimplantation of the right coronary button. Further warm blood cardioplegia is given through the distal end of the graft with partial clamping in order to distend the new aortic root. This maneuver allows assessing hemostasis and valve competence by indirect signs such as good pressure in the root and left ventricular dilatation. A limited echocardiographic view of the aortic valve (AV) may also be obtained at this time. The cardioplegia solution is then slowly aspirated out of the prosthesis without distorting the leaflets for a last check of valve configuration.

\section{Distal closure}

Finally, the two grafts are anastomosed with 5-0 polypropylene. At this time the body temperature is usually back to normal and weaning from $\mathrm{CPB}$ can be started.

\section{Completion}

After weaning from CPB, eventual bleeding is carefully controlled and finally the chest is closed in the standard fashion with steel wires.

\section{Comments}

In certain patients with acute type A aortic dissection, VSRR may be indicated to correct AV dysfunction or to replace a dilated or damaged aortic root. The goal of VSRR is to treat the life-threatening conditions related to the dissection but also to prevent long term complications like recurrent aortic root dissection, false aneurism formation from proximal supra-coronary anastomosis or recurrence of aortic insufficiency (AI). However, as the VSRR procedure increases the cross-clamp time and surgical complexity, it must always be balanced with supra-coronary ascending aorta replacement which is simpler and quicker to perform and which can also correct most of the acute proximal complications like root dissection or AI. Therefore, VSRR should be considered in acute aortic dissection only by surgeons performing those techniques routinely on elective basis.

In acute aortic dissection as well as in aortic root aneurism, we use the VSRR-reimplantation technique because of the maximal support it brings to the weakened tissues in the setting of an aortic wall disease. Also, the hemostatic quality of the reimplantation technique may play an important role in aortic dissection where coagulopathy is frequently present at the end of the surgery. Finally, long-term follow-up has showed excellent stability of the reimplantation technique in many different scenarios including patients with connective tissues disorders and acute aortic dissection.

The factors influencing our decision to perform VSRR in acute type A dissection are therefore the following: aortic root dilatation (Valsalva sinus diameter $>40 \mathrm{~mm}$ ), root dissection, AV quality, presence of AI, presence of acute neurological or vascular complications, patient's age and comorbidities, and connective tissues disorders including bicuspid aortic valve (BAV) syndrome.

VSRR is generally indicated in presence of root dilatation, with or without dissection of the sinuses of Valsalva, in young adult patients ( $<65$ years) without severe comorbidities and without severe acute neurological or vascular complications. However, on the one hand the AV must have leaflets of good quality and on the other hand, the presence of significant $\mathrm{AI}$ is not a contraindication for VSRR.

VSRR can also be indicated in specific patients with type A dissection and normal root size, such as those with connective tissues disorders in whom VSRR should be performed systematically (e.g., Marfan, LoeysDietz syndromes). Patients with AI and dilated aortic annulus $(>28-30 \mathrm{~mm})$ that is frequently seen in case of BAV represent another category of potential candidates for VSRR. In those cases, the VSRR-reimplantation is performed to stabilize the AV repair with its circumferential annuloplasty. Finally, VSRR will also be performed in the rare patients in whom the intimal tear starts or extend into the aortic root. In those cases the reimplantation technique can be used to replace all three Valsalva sinuses although the partial remodeling technique can also be used to replace the sinus with the intimal tear.

Several authors have reported on the results of aortic valve sparing root replacement with the reimplantation technique in the setting of type-A acute dissection (1-4). Dr. David (1) recently reported on his over 25 years experience with the VSSR, including 45 patients with dissection. Although aortic dissection was a significant predictor of death in the long-term, it was not associated with recurrence of aortic regurgitation or reoperation on the valve. Leshnower and associated (2) further reported on 43 consecutive cases treated with the VSRR-reimplantation due to the presence 
of significant root dilatation $(>4.5 \mathrm{~cm})$ or destruction. The mean age of this cohort was $46 \pm 10$ years and, despite a long operation requiring up to 280 minutes of $\mathrm{CPB}$, perioperative mortality was remarkably low at $4.7 \%$. At a mean follow-up of over 3 years, freedom from $2+$ aortic regurgitation and from AV replacement were 94\% and $100 \%$ respectively. Miyahara and colleagues (3) also reported a large series of patients with 'expanded' indication for valve sparing including 21 patients with type A dissection. Although early and mid-term survival in the whole cohort was excellent, type A dissection was associated with a significant risk of reoperation and of recurrence of greater than mild aortic regurgitation over time. Finally a metaanalysis (4) of previous studies on valve preservation and repair techniques in the context of type A dissection showed a low risk of late valve-related bleeding or thromboembolic events at $1.4 \%$ per patient-year and a moderate risk of valve reoperation at $2.1 \%$ per patient-year. However despite this analysis included over 2,400 patients only 59 of them were treated with the reimplantation technique while the vast majority received a simple resuspension of the valve commissures without root replacement.

\section{Conclusions}

In conclusion, aortic VSRR with the reimplantation technique in the context of acute type A aortic dissection can be carried out without increased perioperative risk and is associated with excellent long-term results in terms of recurrence of aortic regurgitation and reoperation on the valve or the aortic root. Nevertheless, experience with the technique as well as careful patient selection are necessary to successfully perform a complex surgery in the context of a life-threatening situation.

\section{Acknowledgements}

None.

\section{Footnote}

Conflicts of Interest: The authors have no conflicts of interest to declare.

\section{References}

1. David TE, Feindel CM, David CM, et al. A quarter of a century of experience with aortic valve-sparing operations. J Thorac Cardiovasc Surg 2014;148:872-9; discussion 879-80.

2. Leshnower BG, Myung RJ, McPherson L, et al. Midterm results of David V valve-sparing aortic root replacement in acute type A aortic dissection. Ann Thorac Surg 2015;99:795-800; discussion 800-1.

3. Miyahara S, Matsueda T, Izawa N, et al. Mid-Term Results of Valve-Sparing Aortic Root Replacement in Patients With Expanded Indications. Ann Thorac Surg 2015;100:845-51; discussion 852.

4. Saczkowski R, Malas T, Mesana T, et al. Aortic valve preservation and repair in acute Type A aortic dissection. Eur J Cardiothorac Surg 2014;45:e220-6.
Cite this article as: Mastrobuoni S, De Kerchove L, Navarra E, Astarci P, Noirhomme P, El Khoury G. Valve sparing-aortic root replacement with the reimplantation technique in acute type A aortic dissection. Ann Cardiothorac Surg 2016;5(4):397400. doi: 10.21037/acs.2016.04.04 